Portland State University

PDXScholar

\title{
Conceptualizing Neighborhood Space: Consistency and Variation of Associations for Neighborhood Factors and Pregnancy Health Across Multiple Neighborhood Units
}

\author{
Lynne C. Messer \\ Portland State University, lynne.messer@pdx.edu \\ Lisa C. Vinikoor-Imler \\ U.S. Environmental Protection Agency \\ Barbara A. Laraia \\ University of California - San Francisco
}

Follow this and additional works at: https://pdxscholar.library.pdx.edu/commhealth_fac

Part of the Community Health and Preventive Medicine Commons, and the Environmental Public Health Commons

Let us know how access to this document benefits you.

\section{Citation Details}

Messer LC, Vinikoor-Imler LC, Laraia BA. Conceptualizing neighborhood space: consistency and variation of associations for neighborhood factors and pregnancy health across multiple neighborhood units. Health and Place, Jul;18(4): 805-13. 2012.

This Article is brought to you for free and open access. It has been accepted for inclusion in Community Health Faculty Publications and Presentations by an authorized administrator of PDXScholar. For more information, please contact pdxscholar@pdx.edu. 


\title{
Conceptualizing neighborhood space: Consistency and variation of associations for neighborhood factors and pregnancy health across multiple neighborhood units
}

\author{
Lynne C. Messer ${ }^{a,}$, Lisa C. Vinikoor-Imler ${ }^{\mathrm{b}, 1}$, and Barbara A. Laraiac,2
}

Lynne C. Messer: lynne.messer@duke.edu; Lisa C. Vinikoor-Imler: vinikorr-imler.lisa@epa.gov; Barbara A. Laraia: laraiab@chc.ucsf.edu

${ }^{a}$ Center for Health Policy and Inequalities Research, Duke Global Health Institute, 2812 Erwin Rd, Suite 403, Durham NC 27705, USA

bNational Center for Environmental Assessment, U.S. Environmental Protection Agency, 109 T.W. Alexander Drive, MD B243-01, Research Triangle Park, NC 27711, USA

'Division of Prevention Sciences, Department of Medicine, Center for Health and Community, University of California, San Francisco, 333 California St, Suite 465, Box 0811, San Francisco, CA 94118, USA

\section{Abstract}

The purpose of this research was to assess the consistency of associations between neighborhood characteristics and pregnancy-related behaviors and outcomes across four nested neighborhood boundaries using race-stratified fixed-slope random-intercept multilevel logistic models. High incivilities was associated with increased smoking, inadequate weight gain and pregnancy-induced hypertension (PIH), while walkability was associated with decreased smoking and PIH for white women across all neighborhood definitions. For African American women, high incivilities was associated with increased smoking and inadequate gestational weight gain, while more walkable neighborhoods appeared protective against smoking and inadequate weight gain in all but the smallest neighborhoods. Associations with neighborhood attributes were similar in effect size across geographies, but less precise as neighborhoods became smaller.

\section{Keywords}

Neighborhood effects; Pregnancy behaviors; Pregnancy outcomes; Estimate precision

\section{Introduction}

Most research considering the influence of neighborhood conditions on health defines neighborhoods using administrative units, including census tracts or block groups (Cubbin et al., 2008; Yen et al., 2009). Authors may note the assumed socio-demographic homogeneity

\footnotetext{
(C) 2012 Elsevier Ltd. All rights reserved.

"Corresponding author: Tel.: +1 919613 5463; fax: +1 9196135466.

1Tel.: +1 919541 2931; fax: +19195415078.

2Tel.: +1 415476 7655; fax: +1 4155021010 .

Competing interests

The authors have no competing interests to declare

Disclaimer

The views expressed in this article are those of the authors and do not necessarily reflect the views or policies of the U.S. EPA.
} 
conferred by various census aggregations but, with few exceptions (Diez-Roux et al., 2001; Krieger et al., 2003), the choice of census unit used to approximate the neighborhood environment is rarely explicitly described.

There are many potential neighborhood characteristics that may be relevant to health. The land use and planning literature, as well as crime and safety literature, can both be employed to guide neighborhood exposure assessment and data collection. From the criminology literature, Perkins et al. (1992) hypothesized that physical evidence of neighborhood degradation, including markers of "physical incivilities" (e.g., litter, graffiti, poor housing conditions), would undermine the confidence that residents have in their neighbors and community, thus increasing the fear of crime and associated stress. Fear of crime and perceived stress have a complex relationship with health, and influence a host of health behaviors including increased smoking, especially among smokers (Cohen and Lichtenstein, 1990; Kouvonen et al., 2005), decreased walking in one's neighborhood and poor eating behaviors (Wardle et al., 2000). From the land use and planning literature, researchers have suggested that providing public space for socializing ("social spaces") and walkable neighborhoods with esthetic qualities and destinations ("walkability") will promote both leisure physical activity and physical activity as a means of transport, which are important for health (Evenson et al., 2009; Frank et al., 2005). To the extent that social spaces and walkability may decrease perceived stress and offer opportunity to walk to nearby destination to purchase food, these constructs could be associated with decreased smoking and healthy eating habits. Based on the cited literature and prior work, the conceptual model underlying this research hypothesized that women are exposed to various neighborhoodlevel health promoting, including walkability and social spaces, and impairing influences, including incivilities. Our conceptual model posited higher levels of physical incivilities would be associated with worse pregnancy-related health behaviors and outcomes because exposure to physical incivilities would result in increased psychosocial stress and may be associated with both individual- and neighborhood-level poverty, which has been associated with adverse pregnancy-related behaviors and outcomes. Conceptually, the model would suggest both walkability and social spaces may be associated with better health behaviors and outcomes. Access to increased social spaces may encourage healthy behaviors (or at least not promote unhealthy behaviors such as smoking), increase the likelihood of social neighborhood-level social interactions during pregnancy and increase perceived social support among expectant women and walkability should facilitate positive maternal health behaviors by providing a safe space within which healthy behaviors may occur.

Estimating neighborhoods with administrative boundaries is challenging is because constructing geographically defined data in the absence of theory or proposed mechanisms can result in the modifiable areal unit problem [MAUP]. The MAUP arises from the imposition of artificial spatial units on continuous geographical conditions; this practice can result in artificial spatial patterns (Heywood et al., 1998; Oliver, 2001) and spurious neighborhood associations. Researchers often fail to account for the degree to which data scale and boundary definitions matter (Sexton et al., 2004).

While generally viewed as a problem, the multiple levels for which geospatial data are available can be seen as a substantive opportunity for exploring the relationships across geographic scales (Subramanian et al., 2001) and testing operational neighborhood definitions. Assessing associations at multiple units of neighborhood aggregation and comparing results across contexts helps establish if the observed association is consistent or may be spurious. Therefore, in this paper, we examine four different neighborhood definitions to consider if the substantive conclusions one would reach about how neighborhood exposures are associated with pregnancy-related behaviors and outcomes varies by the way in which neighborhoods are defined. 


\section{Methods}

\subsection{Study sample}

Over 39,000 birth records from the North Carolina (NC) State Center for Vital Statistics (2001-2005) were obtained for a four county study area in central NC. This analysis was restricted to women delivering singleton infants whose residential addresses could be successfully geocoded to a latitude and longitude within the counties included in the neighborhood audit study area. Of the $98 \%$ of birth files with complete addresses sent for geocoding, approximately $95 \%$ achieved a street-level match. Infants with improbable gestational ages and birthweights $(n=167)$ were excluded (Vinikoor et al., 2011). In addition, due to the small numbers of births to women of other races and ethnicities, only NonHispanic White (white) and non-Hispanic Black (black) women were included in the study. This research was approved by the University of North Carolina's Institutional Review Board.

\subsection{Neighborhood audit study area}

Directly observed data were collected during the summers of 2005 and 2006 on streets within a quarter-mile of the residential addresses of women participating in a cohort study (the Pregnancy, Infection, and Nutrition (PIN3) study). The geographic areas in which PIN3 participants resided were located within, but unevenly distributed across, the four NC counties for which birth records were obtained (Vinikoor et al., 2011). Additional information on the street audit has been reported elsewhere (Evenson et al., 2009).

\subsection{Geographic units of analysis}

Using the directly-observed street-level data, neighborhood-level indices were created at four different units of aggregation ranging from census tracts to tertiary-road networks. County-level road centerline datasets, which are developed and maintained by local county departments and designed to be compatible with county-level tax parcel datasets, were obtained for each of the four counties and merged to construct the study-area road dataset. Details are available elsewhere (Frizelle et al., 2009). Census tract. Census tracts (CT) are small subdivisions of a county (average about 4000 inhabitants) that are designed to be relatively homogenous with respect to population characteristics, socioeconomic status and living conditions. Tract boundaries often follow visible features, such as rivers, but may not follow other administrative (non-visible) boundaries like zip codes (United Census Bureau, 2003). Census block group. Block groups (BG) are smaller geographical units contained within a census tract. They generally house about 1500 people (range 600-3000) (United Census Bureau, 2003). Secondary polygons. Following the approach described by Grannis (1998), secondary polygons (SPs) are smaller units of analysis largely defined by residential street connectivity. The SPs are polygons that are bounded by primary and secondary roads, and by the study area boundary on the outside. Contained within most of these SPs are one or more tertiary roads, which do not cross SP boundaries. By definition, secondary roads are thoroughfares, but they are also high-speed and/or multi-lane and/or divided roads. The SPs were created by first selecting all primary and secondary roads and merging them with the study area boundary using ArcGIS. Where the primary and secondary roads fell just short of intersecting the boundary, they were extended outward until they touched it. Then the merged dataset was converted to polygons and the polygons were enumerated. Tertiary neighborhoods. A tertiary road is defined as a low-speed, residential street that is two-lanes, undivided non-thoroughfares. A tertiary neighborhood (TN), considered one type of functional neighborhood, is a network of tertiary roads that are interconnected and do not cross a primary or secondary road; rather TNs are nested within a SP. The primary and secondary roads that formed the boundary for the TNs were not included as part of the TN because these roads would have to be included in multiple TNs, therefore giving these roads 
undue influence. The SPs and TNs were created in such a way as to ensure that each tertiary road was fully contained within one and only one SP, and SPs were distinct from each other. The street-level data contained within each unit of aggregation were aggregated to that level; for instance, TNs were aggregated to SPs, SPS were aggregatable to census block groups and block groups were aggregatable to census tracts. An example the nested neighborhood definitions can be found in Fig. 1.

In sum, the study area comprised 111 CTs, 317 BGs 1316 SPs and 6159 TNs (Table 1); street segments were rated in 96 (86.5\%), 235 (74.1\%), 497 (37.8\%) and $1369(22.2 \%)$ of these units, respectively.

\subsection{Exposure variables}

Based on prior work (Evenson et al., 2009; Vinikoor et al., 2011), principal components analysis (PCA) applied to audited street-level variables was used to construct indices of three neighborhood-level conditions: physical incivilities, walkability and social spaces. As previously described, "physical incivilities" represents neighborhood disorder, "walkability" represents neighborhood conduciveness for walking and physical activity and "social spaces" characterizes the potential for neighborhood social interactions. Index construction was restricted to those geographic units for which at least $20 \%$ of all street segments were audited, as described elsewhere (Vinikoor et al., 2011). Based on the $20 \%$ restriction, indices were constructed for 62 CTs (55.8\%), 157 BGs (50.0\%), 396 SPs (30.1\%) and 1299 TNs $(21.1 \%)$ in the four county study area. For PCA, each index was constructed using the same variables as previously described (at the block group-level) (Vinikoor et al., 2011) across each neighborhood unit of analysis (Table 1). Neighborhood-specific quartiles (Q1Q4) were constructed for each index; the first quartile (Q1) represented the lowest and the fourth quartile $(\mathrm{Q} 4)$ represented the highest amounts each index. For the walkability index, approximately $50 \%$ of the neighborhoods' component scores were almost identical. Therefore this half of the distribution grouped together, resulting in only three categories for walkability, one representing the first $25 \%$ of the distribution, the second representing the second $25 \%$ of the distribution and the last $50 \%$ of the distribution. Indices at each unit of aggregation were merged with birth records of women residing in the various neighborhood units.

\subsection{Outcome variables}

Using birth certificate data, we examined two pregnancy-related behaviors (smoking and maternal weight gain) and three pregnancy outcomes (pregnancy-induced hypertension/preeclampsia (PIH), term low birthweight (term LBW), and preterm birth (PTB)). These records contained minimal $(<10 \%)$ missing data. Smoking was categorized as present or absent during the pregnancy. Pre-pregnancy weight was not available, but weight gain was categorized as inadequate if a woman gained less than 15 pounds and excessive if a woman gained 40 or more pounds, based on categories used by the Institute of (Institute of Medicine, 1990). PIH was categorized as present or absent during pregnancy, and was not correlated with weight gain among these women $(r=0.06)$. Infants were classified as preterm if their clinically-estimated gestational age was less than 37 weeks' and they weighed less than $3888 \mathrm{~g}$ (Alexander et al., 1996). Term births that weighed less than $2500 \mathrm{~g}$ were classified as term LBW.

\subsection{Statistical analysis}

Descriptive analysis was performed for each level of neighborhood aggregation. Using fixed slope random intercept multilevel models, we examined the associations between the neighborhood-level indices and the pregnancy-related health behaviors and outcomes. Because of suspected heterogeneities in neighborhood effects, odds ratios (ORs) and 95\% 
confidence intervals (CIs) were calculated for both whites and blacks separately at each neighborhood aggregation level. Models were adjusted for maternal age (continuous), maternal education (less than high school, high school, and greater than high school), marital status (not married and married) and parity (no previous pregnancy, one or two previous pregnancies, and three or more previous pregnancies). Analyses were conducted using Stata 10.0 (College Station, Texas). We also calculated the confidence limit ratio (CLR), a ratio of upper $95 \% \mathrm{CI} /$ lower $95 \% \mathrm{CI}$, to allow readers to empirically compare precision across geographic contexts (Poole, 2001). The results presented for the relationships between area indices and maternal health behaviors and outcomes focus more upon the broad trends across geography and outcomes, rather than individual estimates and their statistical significance.

\section{Results}

\subsection{Area description}

Within the neighborhoods for which at least $20 \%$ of the road segments were audited, approximately $51 \%(\mathrm{CT}), 55 \%(\mathrm{BG}), 67 \%(\mathrm{SP})$ and $90 \%(\mathrm{TN})$ of all available roads were audited, on average (Table 1). The median number of roads rated for the TN unit was one (interquartile range: one, seven); $51.5 \%$ of TNs had only one rated road.

\subsection{Index description}

The physical incivilities index was comprised of six variables, and the first principal component explained over $60 \%$ of the total variance at the CT- and BG-levels, and over $40 \%$ at the SP- and TN-levels (Table 2). Social spaces and walkability had four variables each; the percent variance explained by the first component ranged from $71 \%$ (CT) to $42 \%$ (TN) and from $67 \%(\mathrm{CT})$ to $48 \%(\mathrm{TN})$, respectively. For all three indices, the component loadings, and the associated alphas were highest for the larger units of aggregation and became smaller with decreasing neighborhood size, which may result from the index construction optimization procedures, which occurred at the BG-level of aggregation.

\subsection{Sample description}

Most maternal characteristics were consistent across neighborhood units (Table 3). The mean age at pregnancy was about 28.5 years, and between 62 and $65 \%$ of women were white. Slightly more than $7 \%$ gained inadequate gestational weight while just over $26 \%$ gained excessive gestational weight. Over $9 \%$ of women delivered preterm, about $2 \%$ delivered a term LBW infant, and about $5 \%$ had PIH.

\subsection{Physical incivilities, health behaviors and outcomes across residential contexts}

Higher quartiles of physical incivilities were associated with higher odds of smoking and inadequate weight gain for white and black women, across all four neighborhood contexts (Table 4). The associations between incivilities and excessive weight gain among white women differed somewhat depending on the neighborhood unit in which the relationship was modeled, and the associations were generally small and observed differences may result from random error. The relationship between physical incivilities and excessive weight gain was consistently null across neighborhoods for black women.

Higher quartiles of physical incivilities were associated with higher odds of PIH for white women, regardless of the neighborhood unit in which the relationship was assessed, although for TN the association is slightly weaker and the $95 \% \mathrm{CI}$ included the null. The fourth quartile of physical incivilities was associated with higher odds of term LBW and PTB for white women although this association varied across neighborhood units. Among 
black women, there was no association between physical incivilities and any birth outcome across any neighborhood units.

\subsection{Walkability, health behaviors, and outcomes across residential contexts}

Higher quartiles of neighborhood walkability were associated with lower odds of smoking for both white and black women across all neighborhood units (Table 5). A similar inverse relationship was noted between quartiles of walkability and inadequate weight gain for white and black women, but among white women was only statistically associated at the BG-level. The relationship between walkability and excessive weight gain was consistently null for both white and black women regardless of how neighborhoods were defined.

Higher quartiles of walkability were associated with lower odds of PIH for white women with stronger associations noted for the smaller neighborhood contexts, but the no walkability-PIH relationship was apparent for black women. The relationship between higher quartiles of walkability and term LBW was null across the neighborhood units for both white and black women. Null associations were also present for the relationship between quartiles of walkability and PTB, except for an inverse relationship apparent only in the BG unit among white women.

\subsection{Social spaces, health behaviors and outcomes across residential contexts}

The relationship between social spaces and smoking behavior among both black and white women was consistent across neighborhood units; higher quartiles of social spaces were associated with higher odds of smoking, but not all of the associations were statistically significant (Table 6). Across most neighborhood units, higher quartiles of social spaces were associated with higher odds of inadequate weight gain for white women, but this association was less consistently observed for black women. The relationship between social spaces and excessive weight gain was null for both races across neighborhood contexts.

Among white women, higher quartiles of social spaces were associated with higher odds of $\mathrm{PIH}$, but were only statistically significantly associated in models at the SP level; among black women, social spaces was associated with higher odds of PIH only at larger neighborhood contexts (CT). For both white and black women, the relationship between social spaces and term LBW and PTB was null

\section{Discussion}

The three indices, physical incivility, walkability and social spaces, proved to be internally consistent, as indicated by Cronbach's alpha, at larger units of aggregation, but less so in the smaller neighborhoods. While the physical incivilities index alpha remained fairly high across units ( $0.89 \mathrm{CT}$ compared with $0.67 \mathrm{TN})$, suggesting consistency across geographic scale, the walkability and social spaces indices explained $50 \%$ or less of the total variability at the SP and TN level. Lower index alphas at smaller neighborhood units may result from the scales being developed and optimized for the neighborhood BG (Vinikoor et al., 2011). Furthermore, the TNs were usually defined as the single street on which a woman lived, unlike the more densely connected urban road network areas for which this approach was developed (Grannis, 1998); the small number of streets undoubtedly introduced random variability into the $\mathrm{TN}$ indices. While some variability was present, estimate precision, assessed using the CLR, was similar across the neighborhood units.

Among maternal health behaviors, we observed consistent positive results between the physical incivility index and higher odds of smoking and inadequate weight gain across neighborhood contexts for both white and black women, while some evidence for an association with excessive weight gain at CT and BG level was apparent for white women 
only. The robustness of associations across geographic contexts suggests the physical incivilities index was not sensitive to the MAUP for white or black women's health behaviors, and that physical incivilities may be a global marker for negative neighborhood attributes; this finding is consistent with previous work that reported living in a CT with higher levels of physical incivilities was associated with lower odds of engaging in moderate or vigorous physical activity (Laraia et al., 2007). We also found an inverse association for the walkability index with smoking and inadequate weight gain for white and black women, but not at the CT level. Since walking is a common physical activity among pregnant women (Evenson et al., 2004; Evenson and Wen, 2010; Petersen et al., 2005), and walking is likely a local (non-CT) behavior, providing safe walking environments may improve pregnancy-related health. For the social spaces index, fairly consistent associations between the highest quartiles and smoking and inadequate weight gain were noted across contexts for white women, while the estimates for black women were fairly consistently null. These results were unexpected, as social spaces was hypothesized to be a health-promoting neighborhood feature, in part based on prior work that found a positive association between social spaces and adequate pregnancy weight gain (Laraia et al., 2007). This same prior work found social spaces associated with more block group-level poverty, however, which may help explain the observed associations. While social spaces may provide residents a place for sharing and reinforcing social norms, it may also be that not all norms shared in social spaces promote healthy pregnancy-related behavior. Further work developing this construct, and exploring possible urban — rural differences, may be warranted.

Our findings for the associations between neighborhood indices and pregnancy outcomes were less consistent across neighborhood units and varied by race. Some evidence for an association of increased physical incivilities with PIH was noted across three contexts (CT, BG, SP) and a consistent inverse association was observed between higher quartiles of walkability and PIH across three contexts (BG, SP, TN) for white women. Walkability and PTB was inversely associated at the BG level for white women. While there were isolated instances of statistically significant findings, social spaces were not associated with pregnancy outcomes across contexts. Further, no associations between neighborhood indices and pregnancy outcomes were observed for black women. Failing to observe statistically significant results for the associations between neighborhood-level features and adverse birth outcomes among black women is consistent with prior research (Elo et al., 2009; Mason et al., 2009; Messer et al., 2006; O'Campo et al., 2008; Vinikoor et al., 2011) and may result from our models' inability to disentangle the specific neighborhood-level effects of poverty, racial residential segregation, and neighborhood conditions, all of which may be operating simultaneously.

Our use of two census-defined (CT and BG) and two non-census defined neighborhoods (SP and TN) allowed us to assess the utility and consistency of neighborhood indicators across geographic scale, which is an important contribution to the literature. While often critiqued for their seemingly arbitrary boundaries, the findings from these analyses support the use of census-defined neighborhood boundaries for neighborhood-health research. Further, while precision varied somewhat, the conclusions one would draw about neighborhood features and maternal behaviors and outcomes did not vary across contexts, suggesting MAUP is not a substantial concern in this research.

The field of "neighborhood effects" is complicated, in part because neighborhoods both construct and are constructed by the individuals who comprise them; they function as both markers and sources of exposure. For instance, in these analyses, walkability may not be directly related to weight gain or smoking during pregnancy. Rather, observed associations may result from external processes, like selection (non-smokers are attracted to neighborhoods with walkable features) or unmeasured confounding (the social norms 
enforced by residents of walkable neighborhoods may encourage physical activity and weight management, even during pregnancy). It is reasonable to imagine, however, that neighborhoods with walkable features may also prohibit smoking in public places, or other such policies that would discourage smoking in general, in which case walkability serves as a marker for other general health-promoting factors. Further, the relationship between neighborhoods and the individuals residing therein is either reinforced or subverted over time. Teasing apart selection, confounding, temporal and spatial effects would be an impossible undertaking with these data. Therefore, results reported here should not be viewed through the lens of causal effects per se, but rather these indices should be considered as markers of health promoting or damaging environments that are associated with maternal health behaviors and outcomes.

While innovative in its comparison of spatial units, this work is not without limitations. Because TNs were defined as connected tertiary streets bounded by a primary and/or secondary street, a number of primary or secondary streets - and the women who resided upon them - were excluded during construction. The women who lived along primary or secondary roads were somewhat different from the remaining TN sample and this selection, which we assume to be non-differential, may have biased the association between TN indices and maternal health behaviors and outcomes toward the null value. Further, while self-reported smoking may be subject to recall or other bias, smoking in the U.S. South, and $\mathrm{NC}$ in particular, is less-stigmatized than in other regions and women may be more willing to disclose their smoking status. In these analyses, we were unable to adjust for neighborhood socioeconomic status (SES) because these data are not available at small units of aggregation. While this limitation is noteworthy, prior work using similarly constructed indices in another NC county found the correlations between census SES indicators and incivilities and territoriality indices to not be overly high (Laraia et al., 2006). Therefore, neighborhood SES is likely not as substantial confounder to the observed relationships as may be assumed. Additionally, in the absence of pre-pregnancy weight, interpreting gestational weight gain is ambiguous since weight recommendations differ by prepregnancy weight adequacy. If states would record pre-pregnancy weight on birth certificates, significant gains could be made in obesity surveillance, with important public health implications for pregnancy and beyond. Other limitations related to this type of neighborhood-level research have been previously noted (Elo et al., 2009; Mason et al., 2009; Messer et al., 2006; O’Campo et al., 2008; Vinikoor et al., 2011).

A strength of this work is that the large sample size enabled us to conduct racially-stratified analysis at progressively smaller units of analysis. Also, because we collected directlyobserved neighborhood attributes at each unit of aggregation, and these units of aggregation were nested within each other, we were confident that we were comparing similar contexts and nested samples of birth records. A third important strength of this work is that analyses were limited to those neighborhoods for which we audited a sufficient percentage of streets for plausible inference. And last, we restricted the analyses to variables with established validity (Vinikoor et al., 2009).

In this research, we found consistent associations between two neighborhood features, physical incivilities and walkability, and health behaviors, but the relationships with pregnancy outcomes was less regular and varied by maternal race. Associations with black women's outcomes were consistently null, and our inability to detect any neighborhood effect may result from the conflation of race with poverty and poor neighborhood conditions in our study area. While precision was generally lower at the smaller neighborhoods, smaller neighborhood units may function better in more densely connected road networks than found here in the U.S. South. Importantly, we found that census-defined neighborhoods provide comparable information to that obtained from more "local" neighborhoods, 
suggesting that CTs and BGs will continue to have utility for neighborhood-level research. This is an important finding because CTs and BGs in the U.S. South are generally larger and more heterogeneous than more densely populated parts of the country; if CTs and BGs function as appropriate units of aggregation in the South, they should work in other regions as well. That said, because different exposure effects may be more pronounced at different units of aggregation, it remains important to choose the unit of geography that best corresponds to the proposed exposure level and to make sure artificial boundaries are not created that may affect results. Only by using theory or substantive knowledge instead of statistically significant findings to identify the probable unit(s) of effect prior to data collection, variable construction and analyses, can investigators be assured of not constructing area boundaries that lead to spurious associations in neighborhood-effects research.

\section{Acknowledgments}

\section{Funding}

Funding for this study was provided by the Department of Health and Human Services, Health Resources and Services Administration, Maternal \& Child Health Bureau (1 R40MC07841-01-00). Data collection was supported by the National Institutes of Health (NIH)/National Cancer Institute (CA109804). Additional support was provided by the NIH/National Institute of Child Health and Human Development (K01HD047122 and HD37584). The content is solely the responsibility of the authors and does not necessarily represent the official views of the NIH.

The authors would like to thank Kristen Rappazzo and Tom Luben for comments on early versions of the manuscript. We also would like to thank Brian Frizzelle and Kelly Evenson for collaboration on this and other related activities.

\section{References}

Alexander GR, Himes JM, Kaufman RB, Mor J, Kogan M. A United States national reference for fetal growth. Obstetrics and Gynecology. 1996; 87:163-168. [PubMed: 8559516]

United Census Bureau. Census 2000 Summary File 1 Technical Documentation: Appendix A. Census 2000 Geographic Terms and Concepts. 2003

Cohen S, Lichtenstein E. Perceived stress, quitting smoking, and smoking relapse. Health Psychology. 1990; 9:466-478. [PubMed: 2373070]

Cubbin C, Marchi K, Lin M, Bell T, Marshall H, Braveman P. Is neighborhood deprivation independently associated with maternal and infant health? Evidence from Florida and Washington. Maternal and Child Health Journal. 2008; 12:61-74. [PubMed: 17562150]

Diez-Roux AV, Kiefe C, Jacobs D Jr, Haan M, Jackson S, Nieto F, Paton C, Schulz R. Area characteristics and individual-level socioeconomic position indicators in three population-based epidemiologic studies. Annals of Epidemiology. 2001; 11:395-405. [PubMed: 11454499]

Elo I, Culhane J, Kohler I, O’Campo P, Burke J, Messer L, Kaufman J, Laraia B, Eyster J, Holzman C. Neighborhood deprivation and small-for-gestational-age term births among non-Hispanic whites and non-Hispanic blacks in the United States. Paediatric and Perinatal Epidemiology. 2009; 23:8796. [PubMed: 19228318]

Evenson K, Savitz D, Huston S. Leisure-time physical activity among pregnant women in the US. Paediatric and Perinatal Epidemiology. 2004; 18:400-407. [PubMed: 15535815]

Evenson K, Sotres-Alvarez D, Herring A, Messer L, Laraia B, Rodriguez D. Assessing urban and rural neighborhood characteristics using audit and GIS data: derivation and reliability of constructs. International Journal of Behavioral Nutrition and Physical Activity. 2009; 20:16.

Evenson K, Wen F. National trends in self-reported physical activity and sedentary behaviors among pregnant women: NHANES 1999-2006. Preventive Medicine. 2010; 50:123-128. [PubMed: 20053370]

Frank L, Schmid T, Sallis J, Chapman J, Saelens B. Linking objectively measured physical activity with objectively measured urban form. American Journal of Preventive Medicine. 2005; 28:117125. [PubMed: 15694519] 
Frizelle B, Evenson K, Rodriguez D, Laraia B. The importance of accurate road data for spatial applications in public health: customizing a road network. International Journal of Health Geographics. 2009; 8:24. [PubMed: 19409088]

Grannis R. The importance of trivial streets: residential streets and residential segregation. The American Journal of Sociology. 1998; 103:1530-1564.

Heywood, I.; Cornelius, S.; Carver, S. Introduction to Geographical Information Systems. Addison Wesley Longman; New York: 1998.

Kouvonen A, Kivimaki M, Virtanen M, Pentti J, Bahtera J. Work stress, smoking status, and smoking intesity: an observational study of 46,190 employees. Journal of Epidemiology and Community Health. 2005:59.

Krieger N, Chen J, Waterman P, Soobader MJ, Subramanian S, Carson R. Choosing area based socioeconomic measures to monitor social inequalities in low birth weight and childhood lead poisoning: the public health disparities geocoding project (US). Journal of Epidemiology and Community Health. 2003; 57:186-199. [PubMed: 12594195]

Laraia B, Messer L, Evenson K, Kaufman J. Neighborhood factors associated with physical activity and adequacy of weight gain during pregnancy. Journal of Urban Health. 2007; 84:793-806. [PubMed: 17710552]

Laraia BA, Messer LC, Kaufman JS, Dole N, Caughy M, O’Campo P, Savitz DA. Direct observation of neighbourhood attributes in an urban area of the US south. International Journal of Health Geographics. 2006:5. online. [PubMed: 16420702]

Mason S, Messer L, Laraia B, Mendola P. Segregation and preterm birth: the effects of neighborhood racial composition in North Carolina. Health and Place. 2009; 15:1-9. [PubMed: 18359264]

Institute of Medicine. Nutrition during pregnancy. Part I, weight gain. Washington DC: 1990.

Messer L, Kaufman J, Dole N, Savitz D, Laraia B. The neighborhood crime and deprivation environment and preterm birth. Annals of Epidemiology. 2006; 16:455-462. [PubMed: 16290179]

O’Campo P, Burke J, Culhane J, Elo I, Eyster J, Holzman C, Messer L, Kaufman J, Laraia B. Neighborhood deprivation and PTB among non-Hispanic black and non-Hispanic white women in eight geographic areas in the United States. American Journal of Epidemiology. 2008; 167:155163. [PubMed: 17989062]

Oliver L. Shifting boundaries, shifting results: the modifiable areal unit problem. 2001

Perkins D, Meeks J, Taylor RB. The physical environment of street blocks and resident perceptions of crime and disorder: implications for theory and measurement. Journal of Environmental Psychology. 1992; 12:21-34.

Petersen A, Leet T, Brownson R. Correlates of physical activity among pregnant women in the United States. Medicine and Science in Sports and Exercise. 2005; 37:1748-1753. [PubMed: 16260976]

Poole C. Low p-values or narrow confidence intervals: which are more durable. Epidemiology. 2001; 12:291-294. [PubMed: 11337599]

Sexton K, Waller L, McMaster R, Maldonado G, Adgate J. The importance of spatial effects for environmental health policy and research. Human and Ecological Risk Assessment. 2004; 8:109_ 125.

Subramanian S, Duncan C, Jones K. Multilevel perspectives on modeling census data. Environment and Planning. 2001; 33:399-417.

Vinikoor L, Messer L, Evenson K, Laraia B. Neighborhood incivilities, social spaces, walkability, and arterial indices are associated with maternal health behaviors and pregnancy outcomes. Social Science and Medicine. 2011; 73:1302-1311. [PubMed: 21920650]

Vinikoor L, Messer L, Laraia B, Kaufman J. Reliability of variables on the North Carolina birth certificate: a comparison with directly-queried values from a cohort study. Paediatric and Perinatal Epidemiology. 2009; 24:102-112. [PubMed: 20078836]

Wardle J, Steptoeb A, Olivera G, Lipsey Z. Stress, dietary restraint and food intake. Journal of Psychosomatic Research. 2000; 48:195-202. [PubMed: 10719137]

Yen I, Michael Y, Perdue L. Neighborhood environment in studies of health of older adults: a systematic review. American Journal of Preventive Medicine. 2009; 37:455-463. [PubMed: 19840702] 


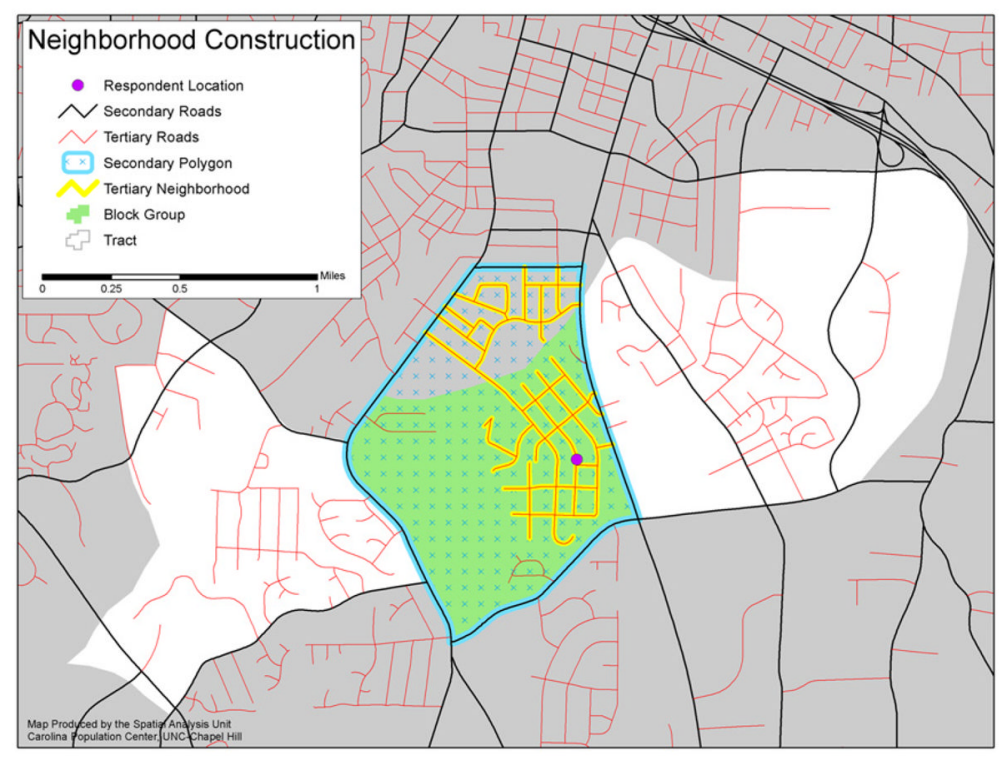

Fig. 1. 


\section{Table 1}

Characteristics of directly-observed neighborhood units; Alamance, Chatham, Durham, Orange Counties, NC (2005 Chatham, Durham, Orange Counties, NC (2006).

\begin{tabular}{|c|c|c|c|c|}
\hline $\begin{array}{l}\text { Four neighborhood contexts: geographic } \\
\text { description }\end{array}$ & Census tract (ct) & Block group (bg) & Secondary polygon (sp) & Tertiary ngbd (tn) \\
\hline Total number of road segments & 111 & 317 & 1316 & 6159 \\
\hline Number with any roads audited & 96 & 235 & 497 & 1369 \\
\hline Number with $\geq 20 \%$ of roads audited & 62 & 157 & 396 & 1299 \\
\hline Mean of roads (SD) & $362.56(262.89)$ & $131.10(76.05)$ & $34.82(49.64)$ & $9.00(20.49)$ \\
\hline Mean audited roads (SD) & $169.73(136.12)$ & $69.69(49.47)$ & $21.70(34.13)$ & $6.83(14.35)$ \\
\hline Mean $\%$ of audited roads & $51.31(22.90)$ & $54.71(24.72)$ & $67.44(27.85)$ & $89.51(21.24)$ \\
\hline Median audited roads & 129 & 54 & 10 & 1 \\
\hline Mean length of roads (miles) (SD) & $49.51(52.40)$ & $16.61(14.04)$ & $3.79(5.57)$ & $0.90(1.93)$ \\
\hline Mean audited roads (miles) (SD) & $18.86(15.23)$ & $7.61(5.45)$ & $2.11(2.96)$ & $0.68(1.26)$ \\
\hline Mean $\%$ of audited road lengths (SD) & $49.3(23.7)$ & $53.4(25.3)$ & $67.1(28.3)$ & $90.44(20.14)$ \\
\hline
\end{tabular}




\section{Table 2}

Principal components analysis loadings and index characteristics across four geographic units; Alamance, Chatham, Durham, Orange Counties, NC (2005 Chatham, Durham, Orange Counties, NC (2006).

\begin{tabular}{|c|c|c|c|c|}
\hline $\begin{array}{l}\text { Principal components analysis:>=20\% } \\
\text { segments rated }\end{array}$ & Census tract (ct) & Block group (bg) & Secondary polygon (sp) & Tertiary ngbd (tn) \\
\hline Number of tracts/groups & 62 & 157 & 396 & 1299 \\
\hline \multicolumn{5}{|l|}{ Physical Inciviliites } \\
\hline Eigenvalue & 3.91 & 3.63 & 2.70 & 2.50 \\
\hline$\%$ variance explained by component 1 & 0.65 & 0.60 & 0.45 & 0.42 \\
\hline Alpha & 0.89 & 0.86 & 0.72 & 0.67 \\
\hline \multicolumn{5}{|l|}{ First principal component loadings: } \\
\hline Fair/poor/deteriorated condition residence & 0.456 & 0.48 & 0.56 & 0.59 \\
\hline Fair/poor condition resident-kept grounds & 0.43 & 0.48 & 0.56 & 0.59 \\
\hline Abandoned/burned/boarded up units & 0.41 & 0.41 & 0.37 & 0.36 \\
\hline Litter & 0.42 & 0.41 & 0.38 & 0.30 \\
\hline Pedestrian-oriented public lighting & -0.29 & -0.23 & -0.23 & -0.18 \\
\hline No trespassing sign & 0.41 & 0.39 & 0.22 & 0.25 \\
\hline \multicolumn{5}{|l|}{ Walkability } \\
\hline Eigenvalue & 2.70 & 2.55 & 2.20 & 1.90 \\
\hline$\%$ variance explained by component 1 & 0.67 & 0.64 & 0.55 & 0.48 \\
\hline Alpha & 0.84 & 0.81 & 0.73 & 0.63 \\
\hline \multicolumn{5}{|l|}{ First principal component loadings: } \\
\hline Neighborhood park/playground & -0.51 & -0.50 & -0.53 & -0.49 \\
\hline Sidewalk in good condition & 0.49 & 0.50 & 0.50 & 0.51 \\
\hline Pedestrian oriented lighted & 0.49 & 0.48 & 0.49 & 0.50 \\
\hline Neighborhood entrance sign & 0.51 & 0.52 & 0.49 & 0.50 \\
\hline \multicolumn{5}{|l|}{ Social spaces } \\
\hline Eigenvalue & 2.85 & 2.30 & 1.97 & 1.67 \\
\hline$\%$ variance explained by component 1 & 0.71 & 0.58 & 0.49 & 0.42 \\
\hline Alpha & 0.86 & 0.74 & 0.51 & 0.39 \\
\hline \multicolumn{5}{|l|}{ First principal component loadings: } \\
\hline Porches in at least half of residences & 0.55 & 0.57 & 0.66 & 0.69 \\
\hline Sidewalk at least one side of the street & -0.53 & -0.51 & -0.38 & -0.27 \\
\hline Traditional lawn or landscaped & 0.53 & 0.56 & 0.65 & 0.68 \\
\hline Visible adult/child & -0.38 & -0.32 & -0.02 & 0.02 \\
\hline
\end{tabular}




\section{Table 3}

Neighborhood-specific individual-level characteristics (Alamance, Chatham, Durham, Orange Counties, NC; 2001-2005).

\begin{tabular}{|c|c|c|c|c|}
\hline & Census tract & Block group & Secondary polygon & Tertiary neighborhood \\
\hline Number of women per context & 23,533 & 23,304 & 21,065 & 17,860 \\
\hline Maternal age in years; mean (SD) & $28.67(6.10)$ & $28.50(6.06)$ & $28.60(6.08)$ & $28.88(6.02)$ \\
\hline \multicolumn{5}{|l|}{ Maternal race } \\
\hline Non-Hispanic White & $15,371(65.32)$ & $14,531(62.35)$ & $13,215(62.73)$ & $11,209(62.76)$ \\
\hline Non-Hispanic African Americans & $8,162(34.68)$ & $8,773(37.65)$ & $7,850(37.27)$ & $6651(37.24)$ \\
\hline \multicolumn{5}{|l|}{ Maternal education $(<\mathrm{HS}, \mathrm{HS},>\mathrm{HS})$} \\
\hline$<\mathrm{HS}$ & $3,063(13.04)$ & $3,083(13.26)$ & $2,663(12.67)$ & $2135(11.98)$ \\
\hline$=\mathrm{HS}$ & $4,588(19.54)$ & 4,630 (19.91) & $4,057(19.30)$ & $3136(17.60)$ \\
\hline$>\mathrm{HS}$ & $15,830(67.42)$ & $15,540(66.83)$ & $14,300(68.03)$ & $12,552(70.43)$ \\
\hline Married=yes (married, not married) & $16,234(68.99)$ & $15,888(68.18)$ & $14,497(68.82)$ & $12,497(69.97)$ \\
\hline \multicolumn{5}{|l|}{ Parity $(0,1-2,3+)$} \\
\hline 0 & $8,285(35.29)$ & $8,258(35.52)$ & $7,525(35.81)$ & $6420(36.04)$ \\
\hline $1-2$ & $11,215(47.77)$ & $11,081(47.67)$ & $9,989(47.54)$ & $8498(47.70)$ \\
\hline $3+$ & $3,976(16.94)$ & $3,907(16.81)$ & 3,497 (16.64) & $2897(16.26)$ \\
\hline Smoker=yes (yes, no) & $2,148(9.16)$ & $2,076(8.94)$ & $1,759(8.38)$ & $1356(7.62)$ \\
\hline \multicolumn{5}{|l|}{ Pregnancy weight gain $(<15,15-39,40+)$} \\
\hline$<15 \mathrm{lbs}$ & $1,710(7.43)$ & $1,753(7.69)$ & $1,556(7.56)$ & $1245(7.14)$ \\
\hline $15-39 \mathrm{lbs}$ & $15,152(65.82)$ & $14,976(65.68)$ & $13,539(65.75)$ & $11,643(66.73)$ \\
\hline $40+\mathrm{lbs}$ & $6,160(26.76)$ & $6,072(26.63)$ & $5,496(26.69)$ & $4561(26.14)$ \\
\hline PIH/Eclampsia=yes (yes, no) & $1,226(5.21)$ & $1,204(5.17)$ & $1,091(5.18)$ & $934(5.23)$ \\
\hline Term low birth weight=yes (yes, no) & $497(2.33)$ & $512(2.43)$ & $457(2.40)$ & $379(2.35)$ \\
\hline Preterm birth=yes (yes, no) & $2,182(9.28)$ & $2,202(9.45)$ & $2,000(9.50)$ & $1702(9.53)$ \\
\hline
\end{tabular}

*All missing data comprise less than $10 \%$ of each variable. 


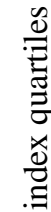

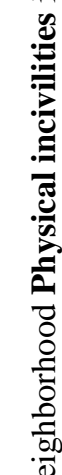

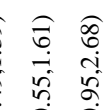

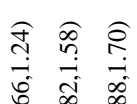

तิ ले ले

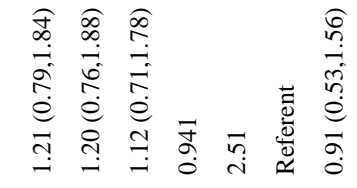

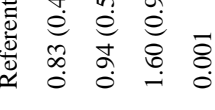

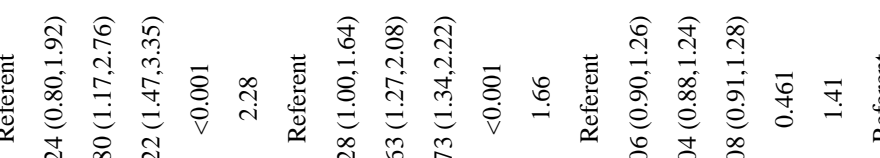

ปั

ํํㄹ

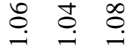

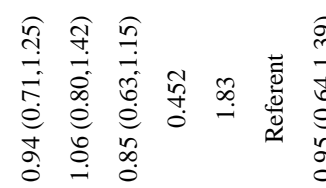

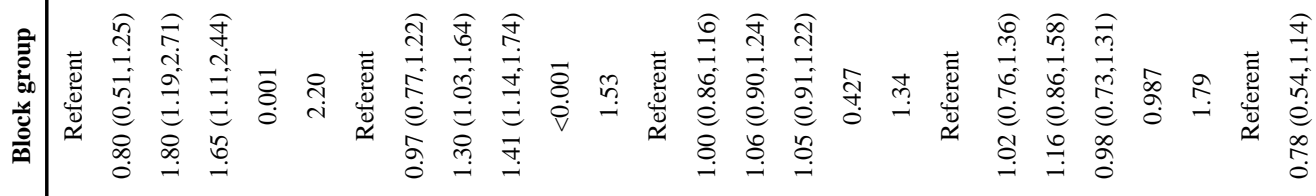

.ี

売

๘

(2)

훙

.

产:

$\Xi$

范

o

त्ञ

.

80

를

ठํํㅇ

ำ

के

雪

吾

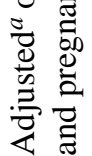

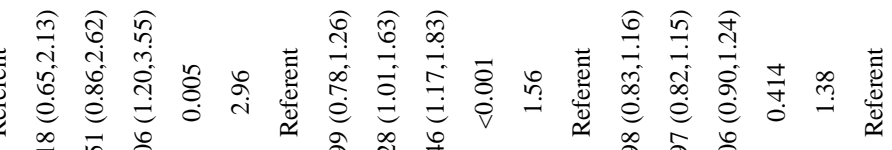

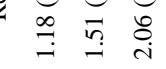

के ঙั

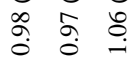

สิ 의

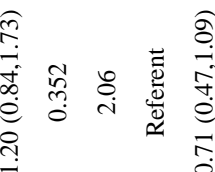

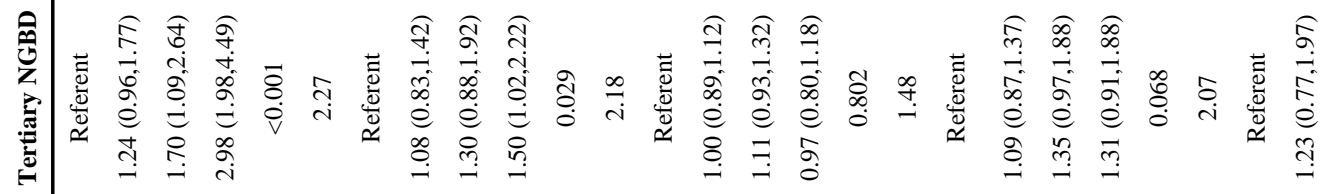

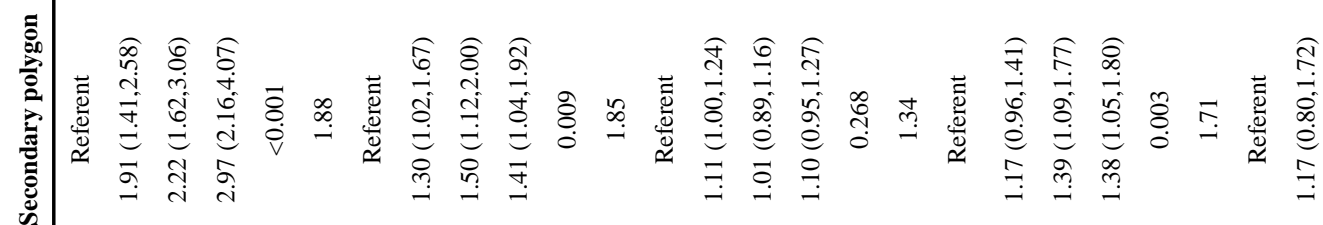

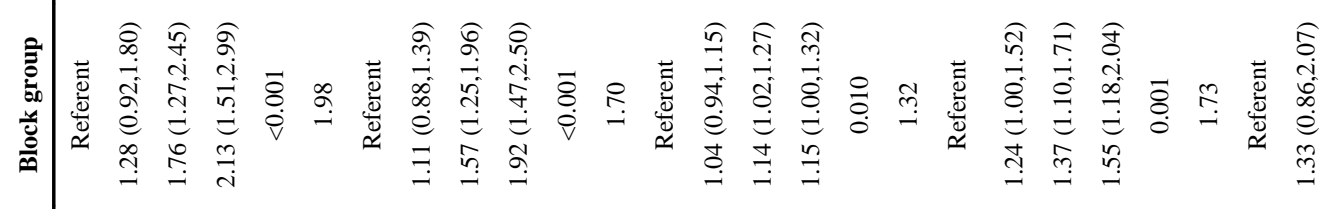

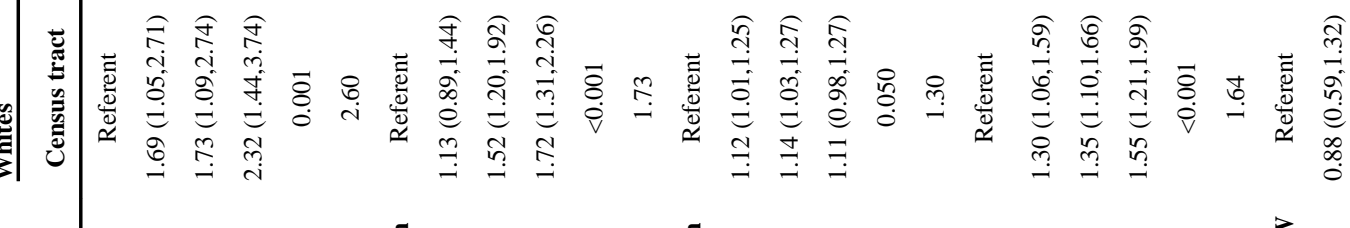

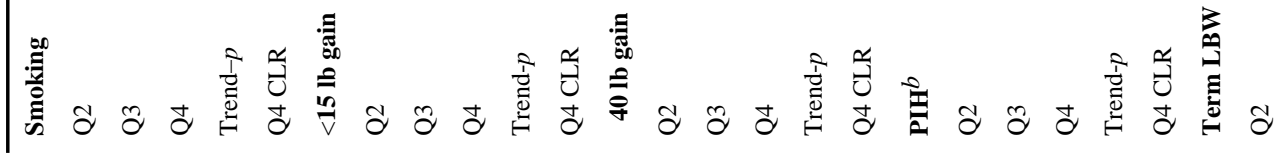




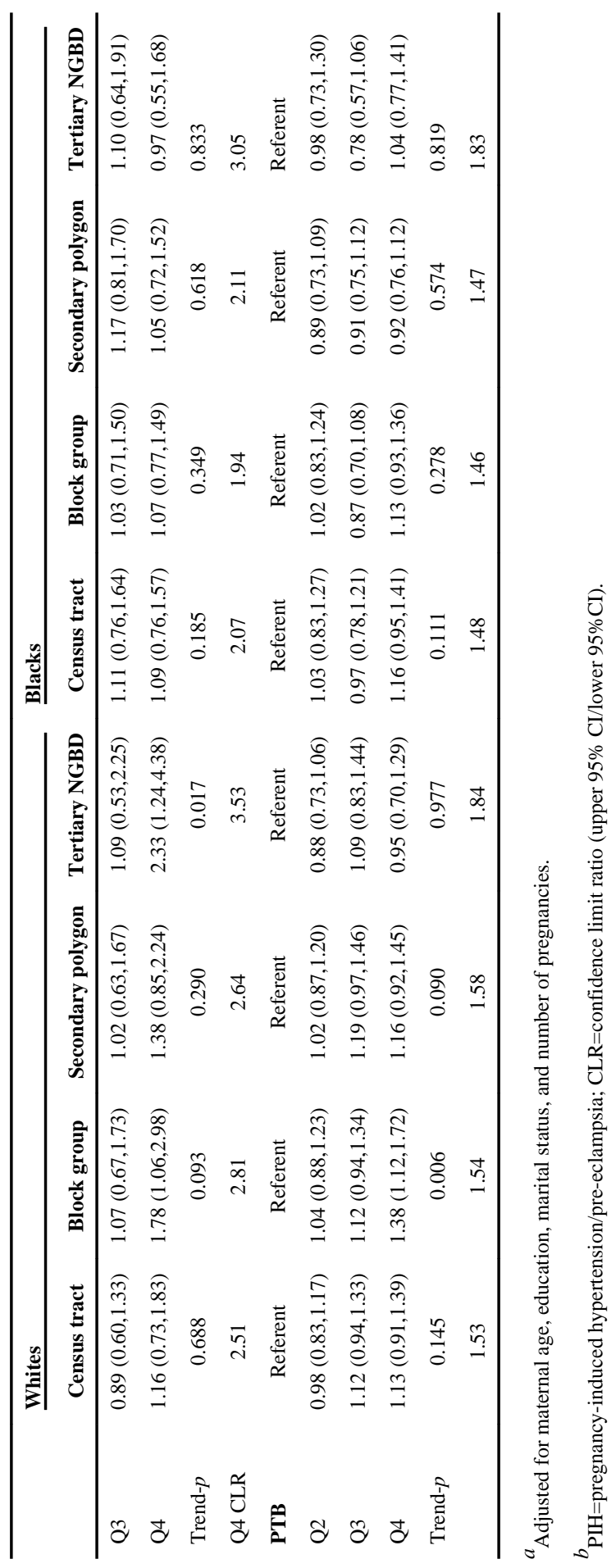




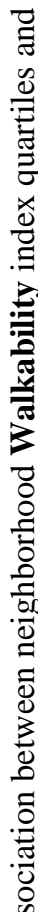

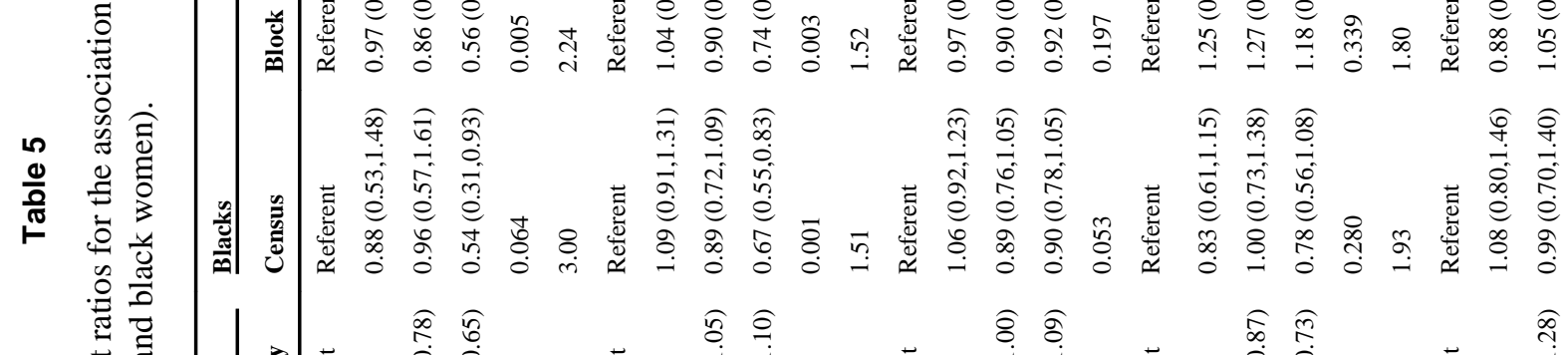

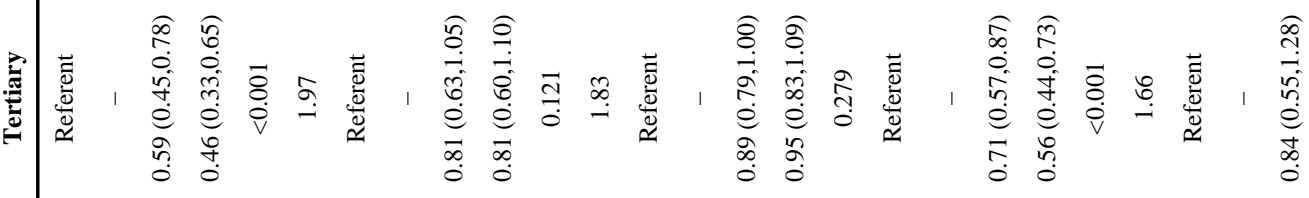

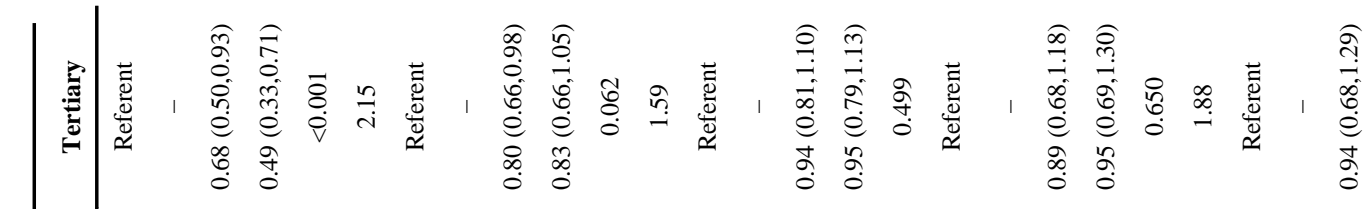

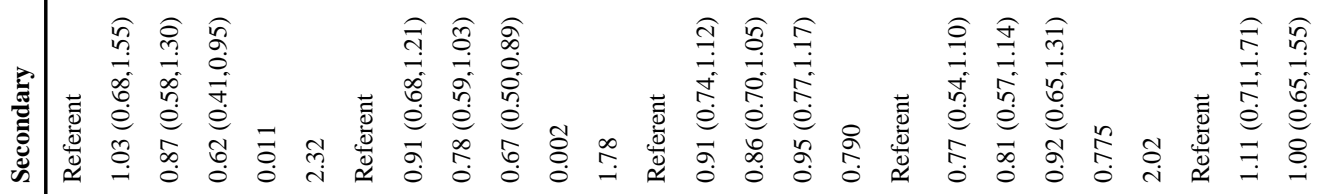

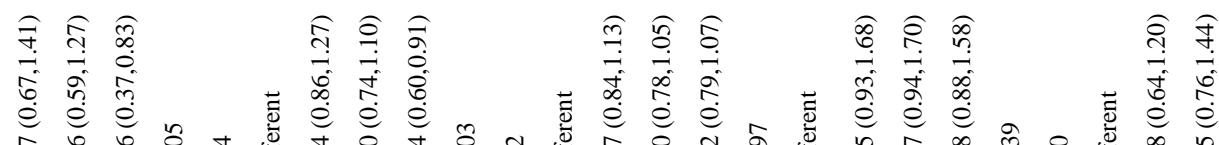

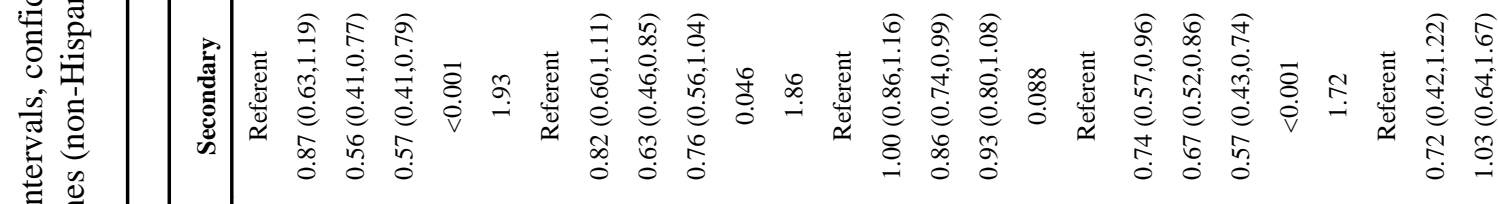




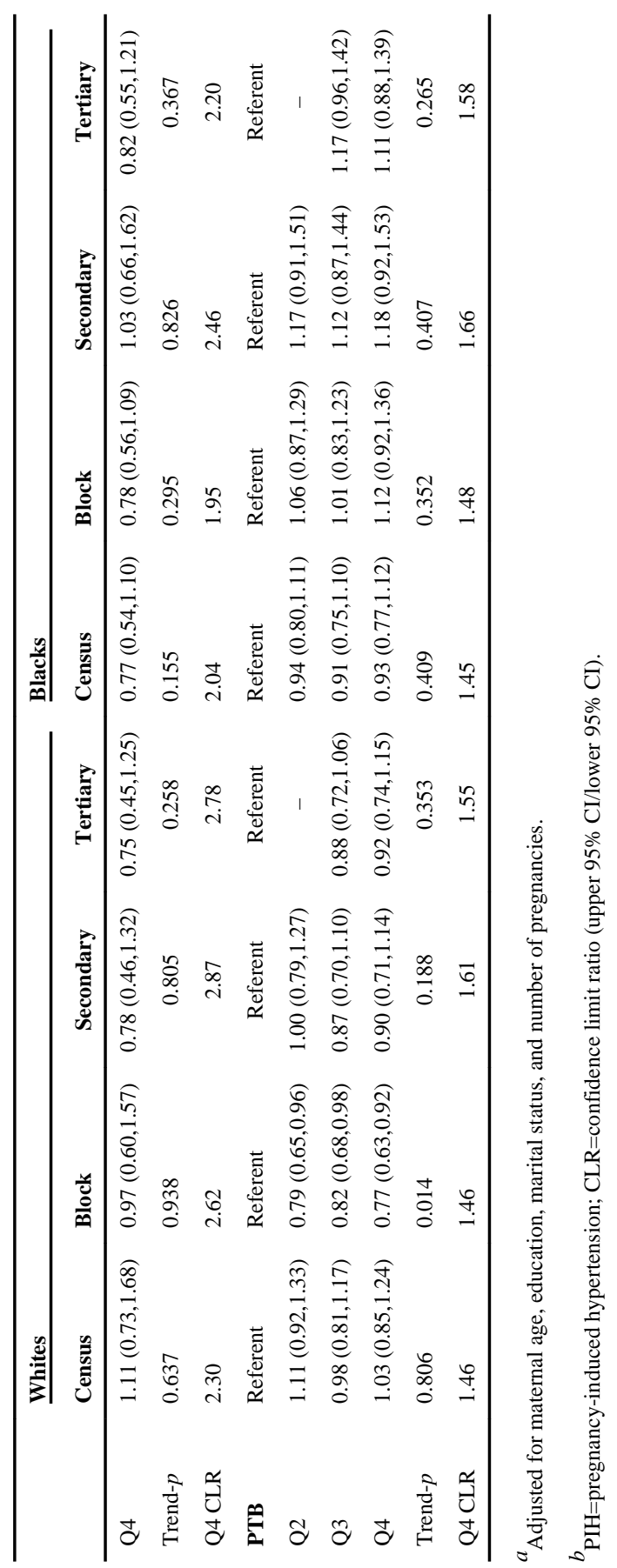




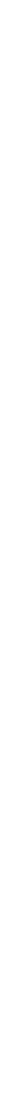

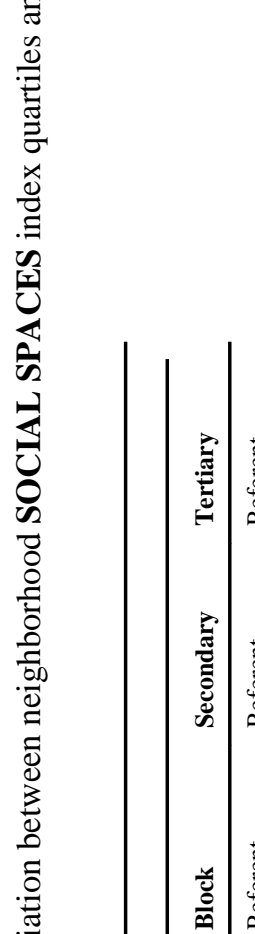

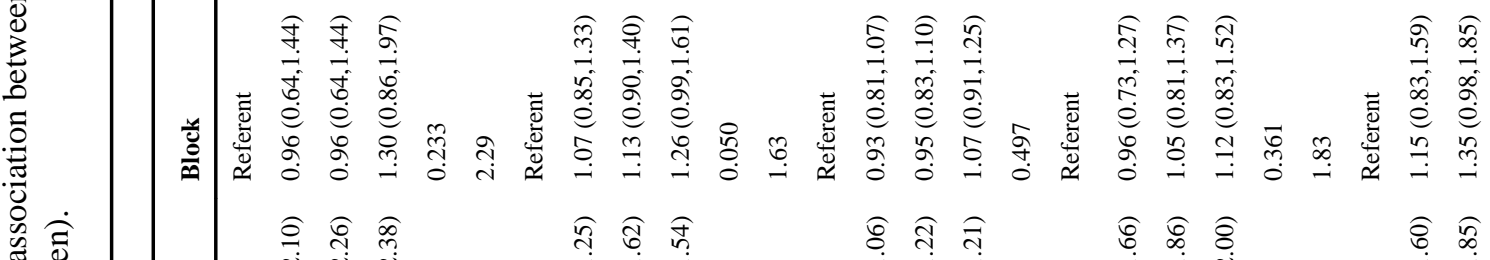

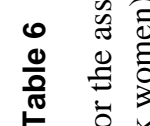

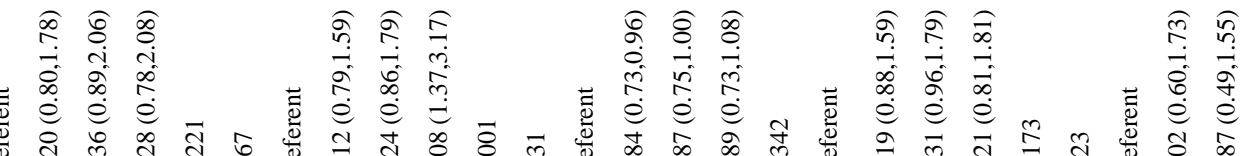

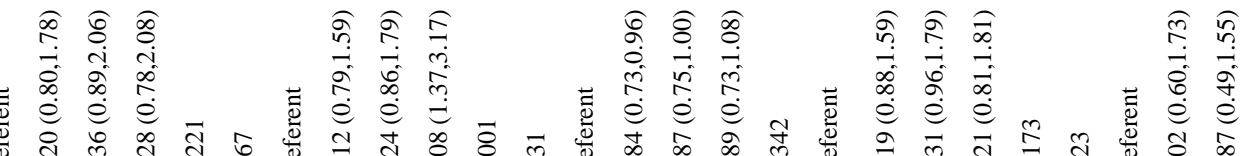

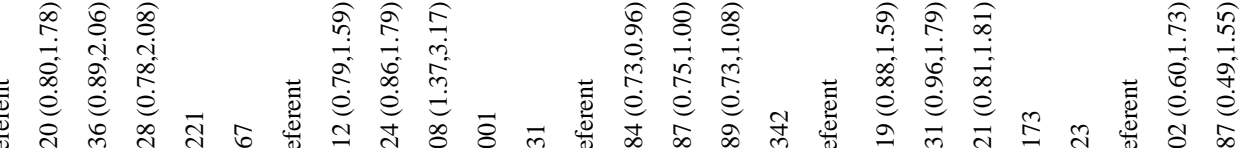

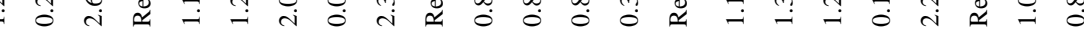

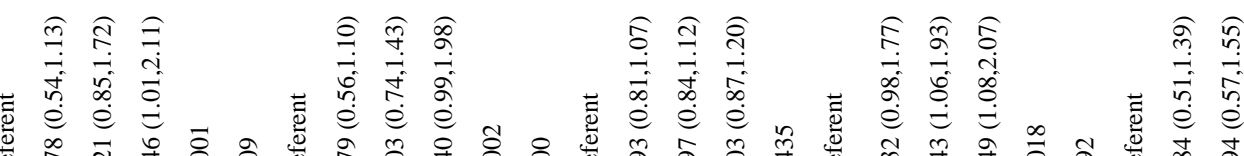

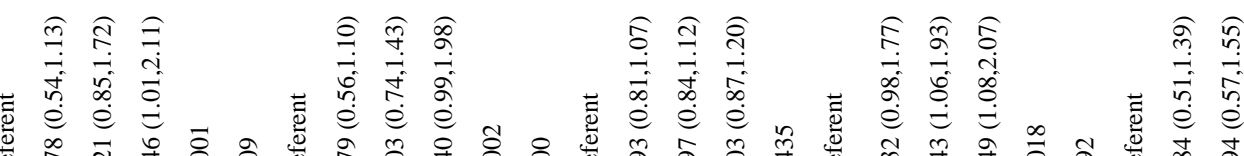

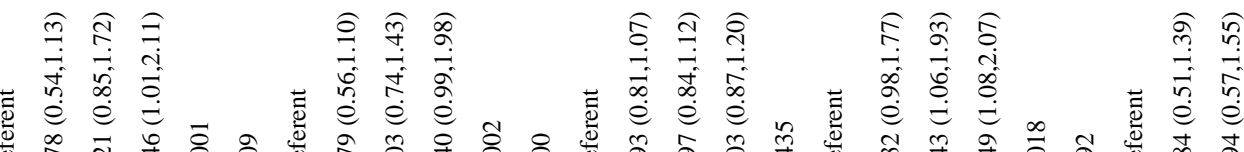

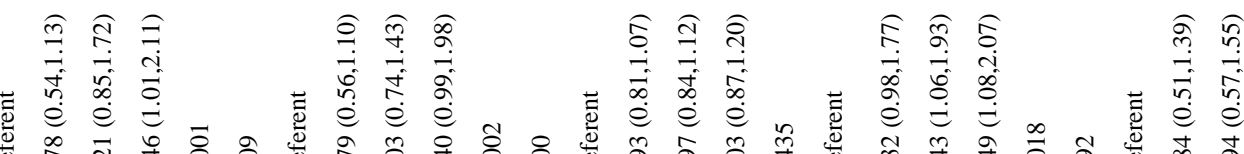

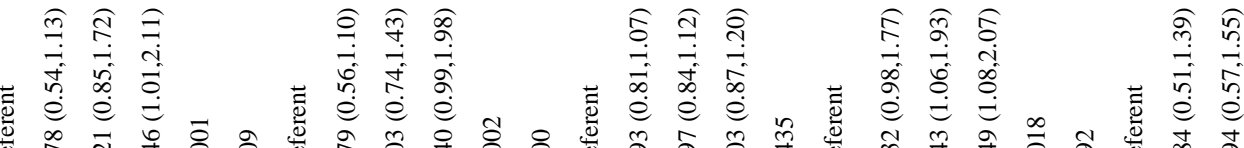

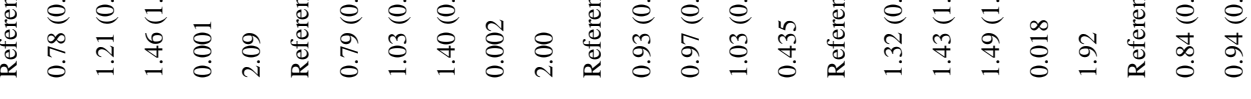

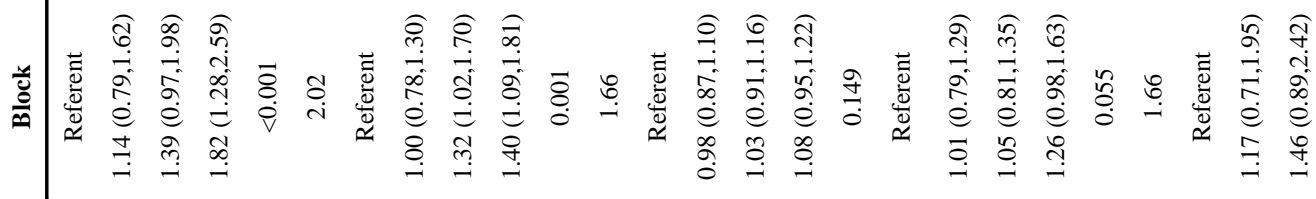

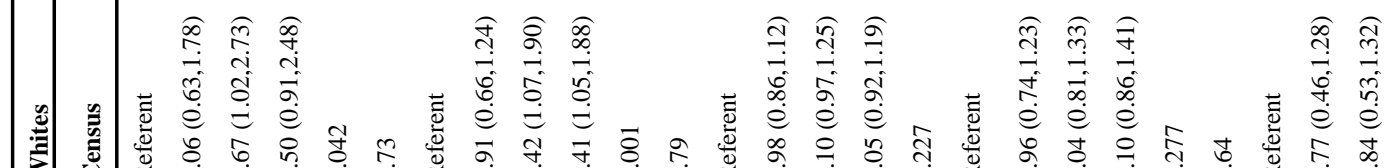
言|

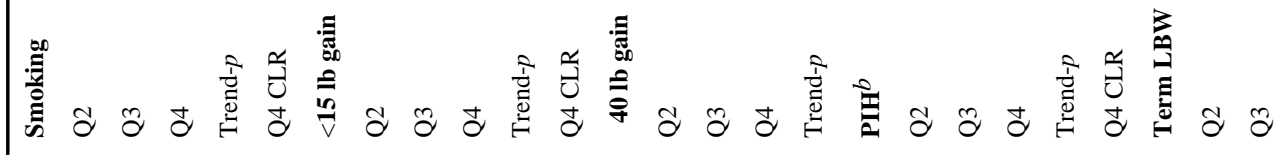




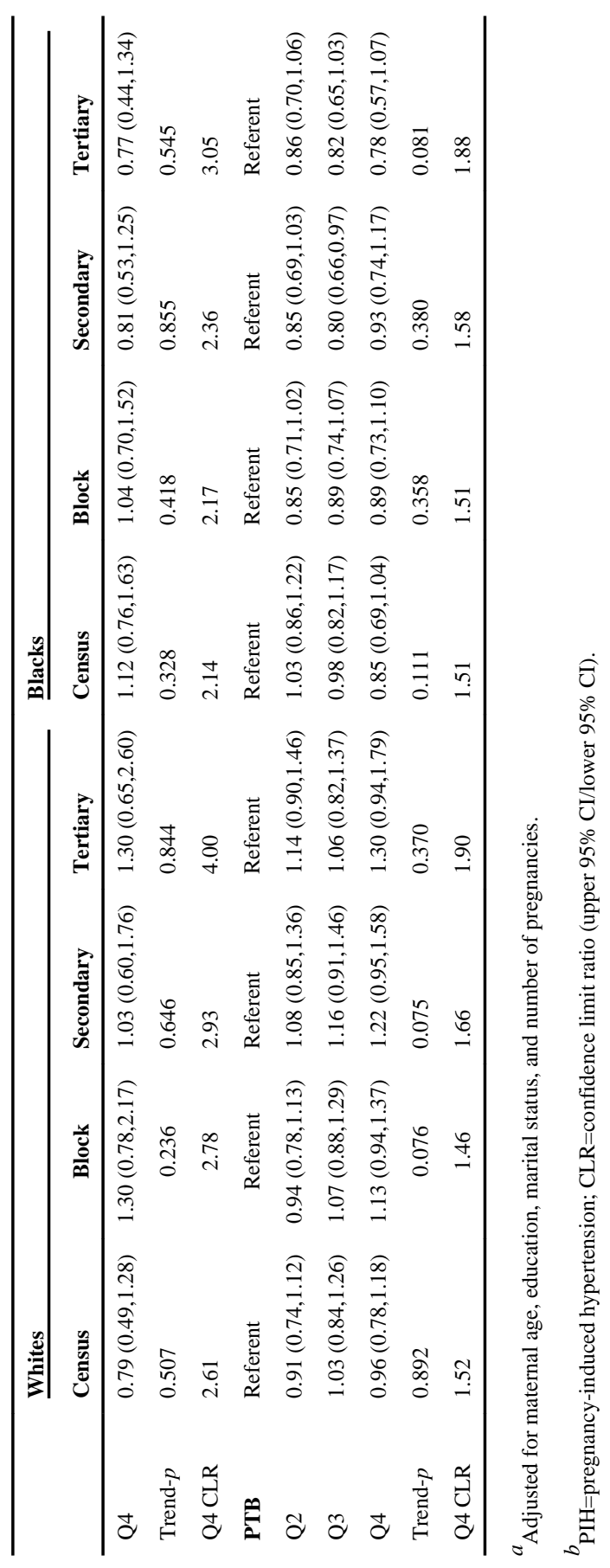

